\title{
Simultaneous Ionization and Excitation of Molecules by Positron Impact
}

\author{
D. A. Cooke, D. J. Murtagh, and G. Laricchia \\ UCL Department of Physics and Astronomy, University College London, Gower Street, London, WC1E 6BT, United Kingdom
}

(Received 11 December 2009; published 19 February 2010)

\begin{abstract}
Cross sections for ionization excitation of molecules by positron impact have been measured for the first time by scattering a positron beam from $\mathrm{CO}_{2}$ and $\mathrm{N}_{2}$. The cross sections have been observed to exceed those for electron impact by up to a factor of $\sim 3$ for $\mathrm{CO}_{2}$ and $\sim 5$ for $\mathrm{N}_{2}$. The enhancement arises primarily via positronium formation. The cross sections account for up to $\sim 12 \%$ and $20 \%$ of the total cross sections for positron scattering from $\mathrm{N}_{2}$ and $\mathrm{CO}_{2}$, respectively.
\end{abstract}

DOI: 10.1103/PhysRevLett.104.073201

PACS numbers: 34.80.Uv, 36.10.Dr

The understanding of positron reactions with atoms and molecules remains a significant challenge, driven by the quest to advance fundamental knowledge of the underlying physical mechanisms, as well as to be able to control and to apply them. In this respect, ionization (including annihilation and Ps formation) is particularly pertinent, with examples ranging from damage limitation to healthy tissue during a scan employing $\beta^{+}$emitters (or indeed targeting of tumors by the same means (e.g., [1]) to the investigation of the interstellar medium (e.g., [2]), and the solar atmosphere (e.g., [3]).

This Letter presents the first measurements of a cross section for positron-induced simultaneous ionization and excitation. $\mathrm{CO}_{2}$ and $\mathrm{N}_{2}$ have been investigated and, in each case, the cross section has been partitioned into direct ionization excitation and positronium (Ps) formation simultaneous with excitation. This work forms part of a broader study of positron-impact ionization phenomena of molecules, the other results of which will be reported elsewhere [4-6]. This study has also been motivated by the earlier observation of considerable $\gamma$-ray-UV photon coincidences following positron impact on $\mathrm{CO}_{2}$ not arising from excited-state Ps formation [7]. The large magnitude of this signal led to the suggestion that it was the result of a channel-coupled mechanism [8,9], a phenomenon which had recently been identified in positron- $\mathrm{O}_{2}$ collisions [10]. It was suggested that the proximity of the thresholds for the $\mathrm{C}$ state of $\mathrm{CO}_{2}$ (reported as $10.56 \mathrm{eV}$ ) and for Ps formation leaving behind $\mathrm{CO}_{2}^{+}\left(A^{2} \Pi_{u}\right)$ (reported as $10.50 \mathrm{eV}$ ) might account for the sizeable coincident signal seen between the $\gamma$ ray from Ps annihilation and the UV photon resulting from the ionic deexcitation.

Details of the present experimental setup can be found in $[11,12]$; those elements specific to this work, along with a brief description, are outlined below and illustrated in Fig. 1. A beam of approximately $10^{4}$ slow positrons $\mathrm{s}^{-1}$, generated by moderating the fast $\beta^{+}$output of a ${ }^{22} \mathrm{Na}$ source with an annealed W-mesh moderator, is constrained radially by a magnetic field of $\sim 10^{-2} \mathrm{~T}$. By applying a positive potential $V_{m}$ to the moderator, the peak energy of the beam can be varied according to $E_{+} \approx e V_{m}+2 \mathrm{eV}$, as established by a retarding-potential analysis. This value is consistent with other determinations for $\mathrm{W}$ under similar non-UHV conditions, including the absolute determinations of $2.4 \pm 0.1 \mathrm{eV}$ [13] and $2.4 \pm 0.3 \mathrm{eV}$ [14] using time-of-flight methods. The transmission of unwanted components, for example, unmoderated positrons or secondary electrons, is substantially reduced by a Wien filter and a cylindrical electrode held at $-500 \mathrm{~V}$, the latter being grounded during measurements with electron projectiles which may be obtained by reversing the polarity of the moderator. In this way, measurements using either positrons or electrons may be taken without substantial modification to the apparatus.

The interaction region consists of a hemispherical polished aluminum gas cell. Ions are extracted from the cell perpendicularly to the beam axis using a lens held at $-500 \mathrm{~V}$ and detected using a channel electron multiplier (CEM) with the cone held at $-2800 \mathrm{~V}$. Opposite this, a photomultiplier tube (PMT) mounted on an extension arm is used to detect low energy (200-600 nm) photons. Light guides coated with $\mathrm{Al}+\mathrm{MgF}_{2}$ line the interior of the extension arm; in conjunction with the polished surface of the gas cell, these serve to enhance photon collection. A borosilicate glass disc may be inserted in front of the PMT

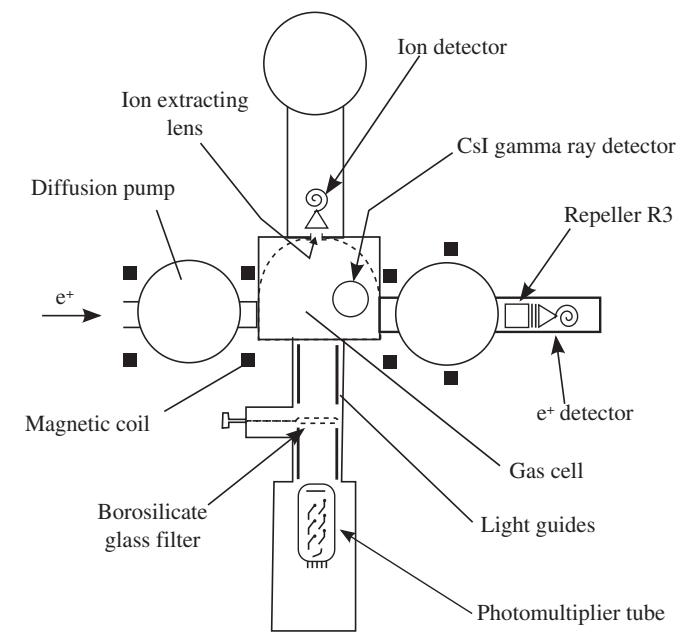

FIG. 1. Interaction region of the beam line showing the positioning of the detectors. 
to block photons with wavelengths shorter than $\sim 280 \mathrm{~nm}$. A photodiode coupled to a CsI scintillation crystal used to detect $\gamma$ rays is positioned above the gas cell. After the interaction region, the intensity of the positron beam is measured by a second CEM.

In the wavelength range of this study, the photons observable following collisions with $\mathrm{CO}_{2}$ arise from the $A^{2} \Pi_{u} \rightarrow X^{2} \Pi g$ and $B^{2} \Sigma_{u}^{+} \rightarrow X^{2} \Pi g$ transitions (293438 and 288-289 nm, respectively [15]), in $\mathrm{CO}_{2}^{+}$, while no transitions in the neutral molecule are reported in the same range $[15,16]$. The possibility of cascade from higher excited states of $\mathrm{CO}_{2}^{+}$may be disregarded as the $C^{2} \Sigma_{g}^{+}$ state is known to be fully predissociative [17] and excitations to higher states are also known to lead to dissociation [18]. Other transitions which may be detectable are $A^{2} \Pi \rightarrow X^{2} \Sigma^{+}$in $\mathrm{CO}^{+}$, formed by the dissociation of $\mathrm{CO}_{2}^{+}$and the $2 P \rightarrow 1 S$ transition from positronium formed in the $2 P$ state. Based, respectively, on electron-impact data [19] and the earlier positron-impact study of [7], both these contributions are expected to be small. For $\mathrm{N}_{2}$, only the $B^{2} \Sigma_{u}^{+} \rightarrow X^{2} \Sigma_{g}^{+}(391.4 \mathrm{~nm})$ transition in $\mathrm{N}_{2}^{+}$is visible together with the photon from the deexcitation of Ps. Unlike the case of $\mathrm{CO}_{2}$, where the contribution from $\operatorname{Ps}(2 P)$ is inseparable from the $B^{2} \Sigma_{u}^{+} \rightarrow X^{2} \Pi g$ transition, in $\mathrm{N}_{2}^{+}$, it can be resolved using the borosilicate glass filter.

The present measurements of the simultaneous ionization excitation cross section $\left(Q_{i}^{\mathrm{ex}}\right)$ were performed by counting coincidences between the PMT and ion detector (henceforth, referred to as "photon-ion" coincidences) on a multichannel analyzer. Similarly, the partitioning of this cross section into contributions from direct ionization $\left(Q_{i}^{\mathrm{ex} /+}\right)$ and positronium formation $\left(Q_{i}^{\mathrm{ex} / \mathrm{Ps}}\right)$ was achieved using $\mathrm{e}^{+}$-photon and $\gamma$-ray-photon coincidences, respectively. These, and the total ionization cross section $\left(Q_{i}^{t}\right)$, were measured simultaneously, ensuring consistent normalization. The lifetimes of the $A^{2} \Pi_{u}$ and $B^{2} \Sigma_{u}^{+}$states of $\mathrm{CO}_{2}^{+}$are $115 \pm 5 \mathrm{~ns}$ and $126 \pm 3 \mathrm{~ns}$, respectively [20], and that of the $B^{2} \Sigma_{u}^{+}$state of $\mathrm{N}_{2}^{+}$is $61.5 \pm 1.1$ ns [21], i.e., less than $1 \%$ of the ion extraction times (typically, $\sim 20 \mu \mathrm{s}$ ), thus resulting in negligible loss of signal.

Generally, a cross section can be derived from a coincidence yield $Y$ (that is, coincidences for the given process per incident positron) via Eq. (1):

$$
Q_{\text {process }}=\frac{1}{n \ell_{\text {eff }}(\text { detection efficiencies })} Y_{\text {process }}
$$

where $n$ is the gas number density measured in the center of the cell and $\ell_{\text {eff }}$ is the effective cell length. The relevant efficiencies depend on which detectors are involved and, for these measurements, the methods employed are outlined below. Full details will be presented elsewhere [4]. The positron detection efficiency of the CEM $\left(\varepsilon_{+}=\right.$ $0.70 \pm 0.07)$ was determined with the aid of an auxiliary detector (CsI) placed in close proximity and by taking the ratio of the number of $\gamma$-ray- $\mathrm{e}^{+}$coincidences $(\mathrm{CsI}+$ $\mathrm{CEM}$ ) and of the $\gamma$-ray counts (CsI) recorded simulta- neously. The ion extraction efficiency $\left(\varepsilon_{\text {ext }}=0.45 \pm\right.$ 0.023 ) was determined by performing a SIMION ${ }^{\mathrm{TM}}$ simulation of the electric field permeating the gas cell with ions created at various positions along the cell while the detection efficiencies for these ions were derived from the work of [22]. The effective (i.e., including geometrical effects) detection efficiency of the CsI $\left[\varepsilon_{\mathrm{CsI}}=(6.24 \pm 0.05) \times\right.$ $10^{-3}$ ] was measured by impinging an $\mathrm{e}^{+}$beam of known intensity upon a retractable annihilation plate. The quantum efficiency of the PMT photocathode as a function of wavelength was supplied by the manufacturer, ET Enterprises Ltd. The solid angle $(\Delta \Omega=0.016 \pm 0.001)$ subtended by the PMT was calculated by considering the active area of the photocathode. The enhancement to this $\left(C_{r}=9.15 \pm 0.13\right)$ due to the internal reflectors was determined by taking the ratio of identical photon-ion coincidence measurements with and without the mirrors. The areal density $n \ell_{\text {eff }}$ was established by the normalization of $Q_{i}^{t}$ for Ar (taken under identical conditions to the present measurements) to that of [23]. The normalization expressed by (1) was carried out via two separate methods: one was to apply the normalization constants determined as described above, the other method was to measure the equivalent electron cross section (where possible) using the same apparatus in order to extract an overall normalization constant and apply it to the positron data after correcting for differences between positron and electron measurements (e.g., in detection efficiencies, in operating pressure-electron measurements having been performed at lower pressures than positrons to retain single-collision conditions). The absolute scales achieved by the two methods have been found to be equal within the total statistical and systematic errors, the latter-amounting up to $(+21,-19) \%$ - being energy independent as arising from the overall uncertainty on the normalization [4]. Measurements were taken with the borosilicate glass filter both in and out to allow the separation of contributions from the $A^{2} \Pi_{u} \rightarrow X^{2} \Pi g$ and $B^{2} \Sigma_{u}^{+} \rightarrow X^{2} \Pi g$ transitions in $\mathrm{CO}_{2}^{+}$, and the separation of $\operatorname{Ps}(2 P)$ from $B^{2} \Sigma_{u}^{+} \rightarrow X^{2} \Sigma_{g}^{+}$ in $\mathrm{N}_{2}^{+}$. A summary of observable transitions for the two glass filter positions is presented in Table I. The mean transmission coefficient through borosilicate glass for each wavelength range was determined from $\mathrm{CO}_{2}^{+}$

TABLE I. Visible transitions for each target gas. With the glass filter inserted, the transmission coefficients are given as premultipliers.

\begin{tabular}{ccc}
\hline \hline & \multicolumn{1}{c}{ Glass filter } \\
Target & In & Out \\
\hline $\mathrm{CO}_{2}$ & $\mathrm{CO}_{2}^{+} 0.85 \times A^{2} \Pi_{u} \rightarrow X^{2} \Pi g$ & $A^{2} \Pi_{u} \rightarrow X^{2} \Pi g$ \\
& $\mathrm{CO}_{2}^{+} 0.22 \times B^{2} \Sigma_{u}^{+} \rightarrow X^{2} \Pi g$ & $B^{2} \Sigma_{u}^{+} \rightarrow X^{2} \Pi g$ \\
& $\mathrm{CO}^{+} 0.90 \times A^{2} \Pi \rightarrow X^{2} \Sigma^{+}$ & $A^{2} \Pi \rightarrow X^{2} \Sigma^{+}$ \\
& $\mathrm{Ps}$ & $2 P \rightarrow 1 S$ \\
$\mathrm{~N}_{2}$ & $0.9 \times B^{2} \Sigma_{u}^{+} \rightarrow X^{2} \Sigma_{g}^{+}$ & $B^{2} \Sigma_{u}^{+} \rightarrow X^{2} \Sigma_{g}^{+}$ \\
& $\mathrm{Ps}$ & $2 P \rightarrow 1 S$ \\
\hline \hline
\end{tabular}


emission-spectra by electron impact [24] and borosilicate transmission curves (e.g., [25]). Note that the $A^{2} \Pi \rightarrow$ $X^{2} \Sigma^{+}$transition in $\mathrm{CO}^{+}$is inseparable from the $A^{2} \Pi_{u} \rightarrow$ $X^{2} \Pi g$ transition in $\mathrm{CO}_{2}^{+}$by this method as the wavelength ranges (300-650 $\mathrm{nm}$ [26]) are almost entirely overlapping, so the transmission coefficients are effectively identical. However, it has been estimated from [19] that this should contribute $<10 \%$ to the overall signal and is henceforth neglected in the present analysis. Separation of states in $\mathrm{CO}_{2}^{+}$may be achieved using simultaneous equations derived from glass-in and glass-out measurements (see Table I for coefficients); for $\mathrm{N}_{2}^{+}$, the glass-in measurement corrected for the transmission coefficient provides the $B^{2} \Sigma_{u}^{+} \rightarrow X^{2} \Sigma_{g}^{+}$yield, while subtracting glass-in from glass-out allows estimation of the yield from Ps $(2 P)$.

Figure 2(a) shows the measured cross section for total ionization (i.e., including Ps formation) simultaneous to ionic excitation $Q_{i}^{\mathrm{ex} / A}$ and $Q_{i}^{\mathrm{ex} / B}$ for positron impact on $\mathrm{CO}_{2}$. Both cross sections peak at an energy significantly lower than corresponding electron cross sections, and ionization into the $A^{2} \Pi_{u}$ state is considerably enhanced over both the equivalent electron-impact cross section and the $B^{2} \Sigma_{u}^{+}$state for positron impact; indeed, at its peak, it is approximately $20 \%$ of the total cross section [27]. Figures 2(b) and 2(c) show, respectively, the partitioning of this cross section into direct ionization and Ps formation components. From these, it is immediately apparent that Ps formation is exclusively responsible for the enhancement of ionization into the $A^{2} \Pi_{u}$ state of $\mathrm{CO}_{2}^{+}$by positron impact. This is corroborative evidence for the hypothesis of [8] (as discussed earlier) concerning Ps formation in $\mathrm{CO}_{2}$ and is consistent with the observations of [28], which identified inner-shell Ps formation using $3 \gamma / 2 \gamma$ ratio measurements.

Figure 2(d) shows a summary of ionization excitation measurements for the $B^{2} \Sigma_{u}^{+} \rightarrow X^{2} \Sigma_{g}^{+}$transition in $\mathrm{N}_{2}^{+}$. In contrast to $\mathrm{CO}_{2}$, the direct ionization process is more important for positrons than electrons-reflecting the situation with ionization without excitation $[4,29]$. However, the excess of $Q_{i}^{\text {ex }}$ for positron impact over that for electron impact (a factor of $\sim 5$ around the peak) is again largely due to Ps formation. At its peak, $Q_{i}^{\text {ex }}$ is approximately $12 \%$ of the corresponding total cross section [27].

For both targets, the contribution from $\operatorname{Ps}(2 P)$ appears to be small, unlike in the noble gases [30]. For $\mathrm{N}_{2}$, it is separable, yielding an estimate for $Q_{\mathrm{Ps}}(2 P)$ of $\sim(1.1 \pm$ 1.1) $\times 10^{-17} \mathrm{~cm}^{2}$, i.e., zero within experimental error. In the case of $\mathrm{CO}_{2}$, it is mixed inseparably, in this work, with the signal from the $B^{2} \Sigma_{u}^{+} \rightarrow X^{2} \Pi g$ transition.

The augmentation of $Q_{i}^{\text {ex }}$ by positron impact over that by electron for these two molecules is clearly dominated by the contribution associated with Ps formation. This is over and above what would be expected from the partitioning of $Q_{i}^{t}$ between Ps formation and direct ionization for the inert atoms [31] and, indeed, these two molecules [6]. As previously noted in [8], in the case of $\mathrm{CO}_{2}$, there is an
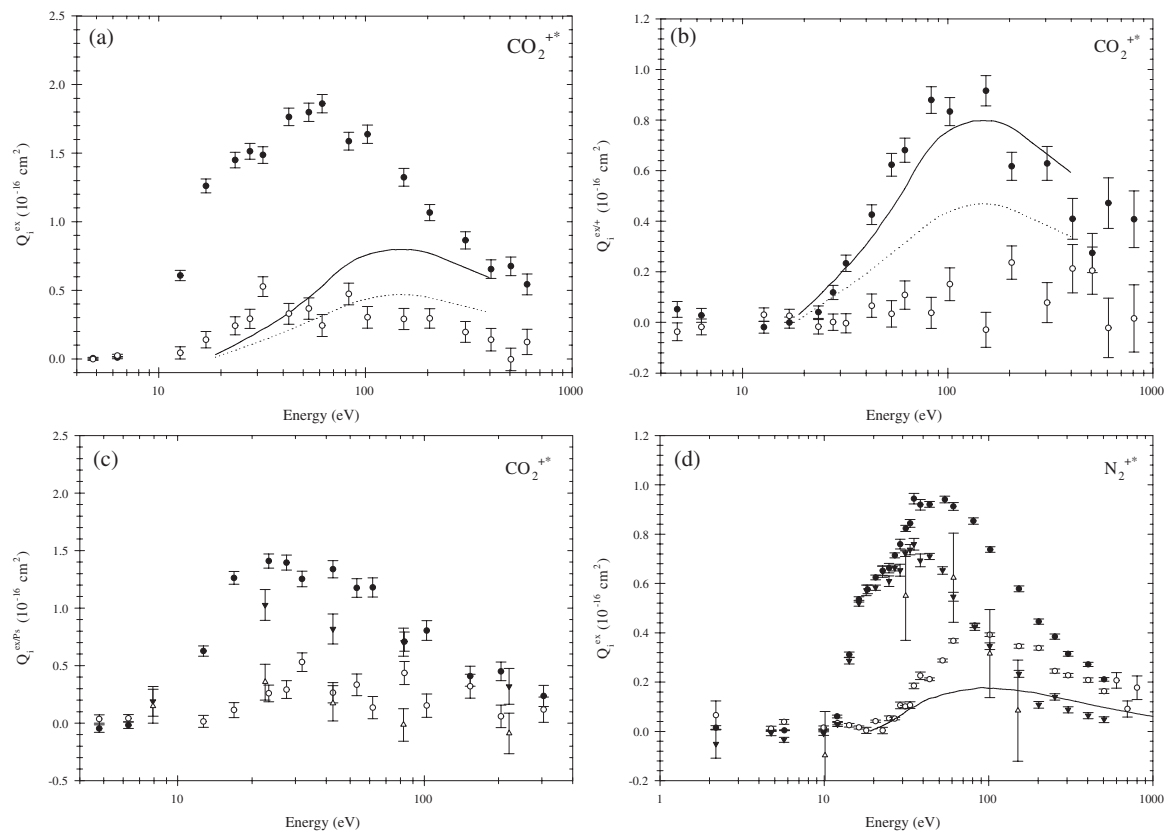

FIG. 2. Present measurements of $Q_{i}^{\text {ex }}$ for $\mathrm{CO}_{2}$ and $\mathrm{N}_{2}$. (a) $Q_{i}^{\text {ex }}$ for the $A^{2} \Pi_{u} \rightarrow X^{2} \Pi_{g}(\bigcirc)$ and $B^{2} \Sigma_{u}^{+} \rightarrow X^{2} \Pi_{g}(\bigcirc)$ transitions in $\mathrm{CO}_{2}^{+}$, compared with equivalent electron measurements of [24] (solid and dotted curves, respectively); (b) as (a), but for direct ionization; (c) as (a), but for Ps formation determined by using two methods: (i) the difference between $Q_{i}^{\text {ex }}$ and $Q_{i}^{\text {ex } /+}({ }$ and $\bigcirc$ ) and (ii) $\gamma$-ray-photon coincidences ( $\nabla$ and $\triangle$ ); (d) $Q_{i}^{\text {ex }}$ for the $B^{2} \Sigma_{u}^{+} \rightarrow X^{2} \Sigma_{g}^{+}$transition in $\mathrm{N}_{2}^{+}$, showing the total (O), contributions from direct ionization $(\bigcirc)$ and Ps formation (determined as in (c) - $\nabla$ and $\triangle$, respectively). The line corresponds to equivalent electron impact results [38]. There is an additional uncertainty of up to $(+21,-19) \%$ on the absolute scale of the present measurements due to the errors on the normalization. 
extremely close energetic proximity for the excitation of the ${ }^{1} \Sigma_{u}^{+}$neutral state $\{10.30-11.28 \mathrm{eV}$ (see, e.g., [32,33]) and the $A^{2} \Pi_{u}$ ionic state formed by Ps formation (10.52$11.22 \mathrm{eV}$, based on an ionization potential of $13.78 \mathrm{eV}$ [34] and vibrational level assignments of $[15,24])$. Also, from the potential energy curves for $\mathrm{N}_{2}$ [35], it is possible to deduce that a similar near degeneracy may exist between a number of neutral excited states $\left(b^{1} \Pi_{u}, b^{\prime 1} \Sigma_{u}^{+}, c^{1} \Pi_{u}\right.$, and $c^{\prime 1} \Sigma_{u}^{+}$, over the energy range $12.50-13.95 \mathrm{eV}$ [36]) and the ionic $B^{2} \Sigma_{u}^{+}$state produced via Ps formation (at approximately $12.0 \mathrm{eV}$ ). Thus, for both targets, the enhancement of $Q_{i}^{\text {ex }}$ might result from the coupling between two quasidegenerate channels: one the excitation of the neutral, the other of an ionic state induced by Ps formation. Indeed, since formation of ground state Ps lowers the ionic energy thresholds by $6.8 \mathrm{eV}$, an amount close to typical energy differences between excited molecular and ionic states, the "accidental resonance" between these excitations is likely to be a common feature of molecular ionization by positronium formation - the near-degeneracy made probable (in contrast with atoms) by the variation of the molecular interaction energy with internuclear distance and the fine energy-structure associated with vibrational and rotational excitations.

In addition to providing a hypothesis for the enhancement of the simultaneous Ps formation-excitation cross section, the accidental resonance described above may provide an explanation for the comparative dearth in $\mathrm{N}_{2}$ of Ps formation in excited states, as corresponding binding energies $(1.7 \mathrm{eV}$ for $n=2)$ are too small to result in significant overlap of molecular states.

In conclusion, ionization excitation of $\mathrm{CO}_{2}$ and $\mathrm{N}_{2}$ is a more significant process for positron impact than for electrons. An examination of the potential energy curves ([35], for $\mathrm{N}_{2}$ ) or energy levels (e.g., $[33,37]$, for $\mathrm{CO}_{2}$ ) for these molecules suggests that the positron may be able to excite the molecules quasiresonantly by coupling excited neutral and ionic states through Ps formation and, perhaps, allow the system to relax to the slightly lower energy configuration formed by Ps and an excited ionic state. Further experimental and theoretical work is anticipated.

We wish to thank Ann Orel and Jonathan Tennyson for useful discussions, Rafid Jawad and John Dumper for expert technical assistance, the Engineering and Physical Sciences Research Council for supporting this research.

[1] V. Caridad et al., Cancer Biotherapy and Radiopharm. 23, 371 (2008).

[2] N. Guessoum, P. Jean, and W. Gillard, Astron. Astrophys. 436, 171 (2005).

[3] R. J. Murphy, G. H. Share, J. G. Skibo, and B. Kozlovsky, Astrophys. J. Suppl. Ser. 161, 495 (2005).

[4] D. A. Cooke, D. J. Murtagh, and G. Laricchia (to be published).

[5] D. A. Cooke, D. J. Murtagh, and G. Laricchia, J. Phys. Conf. Ser. (to be published).
[6] G. Laricchia et al., J. Phys. Conf. Ser. 194, 012036 (2009).

[7] G. Laricchia, M. Charlton, and T. C. Griffiths, J. Phys. B 21, L227 (1988).

[8] G. Laricchia and J. Moxom, Phys. Lett. A 174, 255 (1993).

[9] G. Laricchia, J. Moxom, M. Charlton, Á. Kövér, and W. E. Meyerhof, Hyperfine Interact. 89, 209 (1994).

[10] G. Laricchia, J. Moxom, and M. Charlton, Phys. Rev. Lett. 70, 3229 (1993).

[11] D. A. Cooke, D. J. Murtagh, À. Kövér, and G. Laricchia, Nucl. Instrum. Methods Phys. Res., Sect. B 266, 466 (2008).

[12] M. Szłuińska, P. Van Reeth, and G. Laricchia, Nucl. Instrum. Methods Phys. Res., Sect. B 192, 215 (2002).

[13] V. Kara, Ph.D. thesis, University College London, 1999.

[14] M. Szłuińska and G. Laricchia, Nucl. Instrum. Methods Phys. Res., Sect. B 221, 100 (2004).

[15] J. M. Ajello, J. Chem. Phys. 55, 3169 (1971).

[16] M. Monce, Phys. Rev. A 38, 3351 (1988).

[17] J. H. D. Eland, Int. J. Mass Spectrom. Ion Phys. 9, 397 (1972).

[18] J. H. D. Eland and J. Berkowitz, J. Chem. Phys. 67, 2782 (1977).

[19] K. Furuya, A. Matsuo, and T. Ogawa, J. Phys. B 35, 3077 (2002).

[20] C. Herran, F. Arqueros, and J. Campos, J. Mol. Spectrosc. 97, 244 (1983).

[21] F. Remy and M. A. Dumont, J. Quant. Spectrosc. Radiat. Transfer 20, 217 (1978).

[22] M. Krems, J. Zirbel, M. Thomason, and R. D. DuBois, Rev. Sci. Instrum. 76, 093305 (2005).

[23] G. Laricchia, P. Van Reeth, M. Szłuińska, and J. Moxom, J. Phys. B 35, 2525 (2002).

[24] S. Tsurubuchi and T. Iwai, J. Phys. Soc. Jpn. 37, 1077 (1974).

[25] http://www.atscope.com.au/photometry.html, 2009.

[26] J. M. Ajello, J. Chem. Phys. 55, 3158 (1971).

[27] W. E. Kauppila and T. S. Stein, Adv. At. Mol. Opt. Phys. 26, 1 (1990).

[28] W.E. Kauppila, E. G. Miller, H.F. M. Mohamed, K. Pipinos, T. S. Stein, and E. Surdutovich, Phys. Rev. Lett. 93, 113401 (2004).

[29] H. Bluhme et al., J. Phys. B 31, 4631 (1998).

[30] D. J. Murtagh, D. A. Cooke, and G. Laricchia, Phys. Rev. Lett. 102, 133202 (2009).

[31] G. Laricchia, S. Armitage, Á. Kövér, and D. J. Murtagh, Adv. At. Mol. Opt. Phys. 56, 1 (2008).

[32] H. Nakatsuji, Chem. Phys. 75, 425 (1983).

[33] W. F. Chan, G. Cooper, and C. E. Brion, Chem. Phys. 178, 401, 1993.

[34] NIST Chemistry Web-Book, NIST Standard Reference Database Number 69, edited by P. J. Linstrom and W. G. Mallard (National Institute of Standards and Technology, Gaithersburg, MD, 2005).

[35] A. Lofthus and P. H. Krupenie, J. Phys. Chem. Ref. Data 6, 113 (1977)

[36] W.F. Chan, G. Cooper, R. N. S. Sodhi, and C. E. Brion, Chem. Phys. 170, 81 (1993).

[37] R. J. Buenker, M. Honigmann, H-P. Liebermann, and M. Kimura, J. Chem. Phys. 113, 1046 (2000).

[38] W. L. Borst and E. C. Zipf, Phys. Rev. A 1, 834 (1970). 